\title{
Analysis of Factors Contributing to Repeat Surgery in Multi-Segments Cervical Ossification of Posterior Longi- tudinal Ligament
}

\author{
Ikchan Jeon, M.D., Y Yong Eun Cho, M.D., Ph.D. ${ }^{2}$ \\ Department of Neurosurgery, ${ }^{1}$ Yeungnam University Hospital, Yeungnam University College of Medicine, Daegu, Korea \\ Department of Neurosurgery, ${ }^{2}$ Gangnam Severance Hospital, The Spinal and Spinal Cord Institute, Yonsei Univer sity College of Medicine, \\ Seoul, Korea
}

Objective : Cervical ossification of the posterior longitudinal ligament (OPLL) can be treated via anterior or posterior approach, or both. The optimal approach depends on the characteristics of OPLL and cervical curvature. Although most patients can be successfully treated by a single surgery with the proper approach, renewed or newly developed neurological deterioration often requires repeat surgery.

Methods : Twenty-seven patients with renewed or newly developed neurological deterioration requiring salvage surgery for multi-segment cervical OPLL were enrolled. Ten patients (group AP) underwent anterior approach, and 17 patients (group PA) underwent posterior approach at the initial surgery. Clinical and radiological data from initial and repeat surgeries were obtained and analyzed retrospectively.

Results : The intervals between the initial and repeat surgeries were $102.80 \pm 60.08$ months (group AP) and $61.00 \pm 8.16$ months (group PA) $(p<0.05)$. In group AP, the main OPLL lesions were removed during the initial surgery. There was a tendency that the site of main OPLL lesions causing renewed or newly developed neurological deterioration were different from that of the initial surgery $(8 / 10, p<0.05)$. Repeat surgery was performed for progressed OPLL lesions at another segment as the main pathology. In group $\mathrm{PA}$, the main OPLL lesions at the initial surgery continued as the main pathology for repeat surgery. Progression of kyphosis in the cervical curvature (Cobb's angle on C2-7 and segmental angle on the main OPLL lesion) was noted between the initial and repeat surgeries. Group PA showed more kyphotic cervical curvature compared to group AP at the time of repeat surgery $(p<0.05)$.

Conclusion : The reasons for repeat surgery depend on the type of initial surgery. The main factors leading to repeat surgery are progression of remnant OPLL at a different segment in group AP and kyphotic change of the cervical curvature in group PA.

Key Words : Cervical vertebrae · Ossification of posterior longitudinal ligament $\cdot$ Reoperation · Kyphosis.

\footnotetext{
- Received : January 5, 2017 •Revised : April 20, 2017 •Accepted : June 23, 2017

- Address for reprints : Yong Eun Cho, M.D., Ph.D.

Department of Neurosurgery, Gangnam Severance Hospital, The Spinal and Spinal Cord Institute, Yonsei University College of Medicine, 211 Eonju-ro, Gangnam-gu, Seoul 06273, Korea

Tel : +82-2-2019-3390, Fax : +82-2-2019-3840, E-mail : yecho@yuhs.ac
}

This is an Open Access article distributed under the terms of the Creative Commons Attribution Non-Commercial License (http://creativecommons.org/licenses/by-nc/4.0) which permits unrestricted non-commercial use, distribution, and reproduction in any medium, provided the original work is properly cited. 


\section{INTRODUCTION}

Ossification of the posterior longitudinal ligament (OPLL) is characterized by the replacement of ligamentous tissue with new ectopic bone formation, potentially causing spinal cord compression and progressive neurological deterioration such as cervical myelopathy and/or radiculopathy ${ }^{10)}$. Although patients presenting with mild symptoms can be treated non-surgically, surgical decompression is recommended for advanced or deteriorating prominent neurological symptoms ${ }^{1)}$. OPLL can be treated by anterior or posterior decompression, or both, the optimal surgical approach depend on OPLL characteristics, the sagittal alignment of the cervical spine, the severity of stenosis, and history of previous cervical surgery ${ }^{1,13,14)}$.

Most of cervical OPLL involves multi-segments. Multi-segment cervical OPLL can be usually treated by a single surgery with a suitable anterior or posterior approach, even though OPLL lesions which are related or not with neurological symptom are remained. However, renewed or newly developed neurological deterioration often makes later repeat surgery necessary. There are not sufficient literatures discussing about these problems so far. In this study, we discussed about the mechanism and causative factors related with renewed or newly developed neurological deterioration requiring repeat surgery depending on the type of previous surgery in multi-segments OPLL.

\section{MATERIALS AND METHODS}

\section{Patient population}

From 1996 through 2012, 27 patients (22 males and five females, mean age $61.26 \pm 7.37$ years) with cervical OPLL span- ning more than three segments and renewed or newly developed neurological deterioration requiring repeat surgery after initial anterior or posterior decompression surgery were enrolled. Patients were excluded from this study that had history of other surgery, trauma, infection, tumor, congenital disorders in cervical spine, or neurological deficits related to other portions of the spine, limbs, or brain that could affect or confuse evaluation of neurological status associated with the cervical condition.

The patients underwent only anterior or posterior surgery at the initial surgery, depending on the surgeon's decision in our and other hospitals. Sufficient neurological and functional recovery after the initial surgery did not require immediate additional surgery. Ten patients (group AP; nine males and one female, mean age $61.70 \pm 6.18$ years) were approached anteriorly (two of anterior cervical corpectomy and fusion [ACCF], eight of anterior cervical discectomy and fusion [ACDF]) during the initial surgery, and underwent repeat surgery via the posterior approach (seven of decompressive laminectomy plus fusion with screw fixation, three of laminoplasty). Seventeen patients (group PA; 13 males and four females, mean age $61.00 \pm 8.15$ years) received initial surgery with a posterior approach (11 of decompressive laminectomy alone [subgroup LN], five of laminoplasty [subgroup LP], and one of decompressive laminectomy plus fusion with screw fixation), and underwent repeat surgery via the anterior approach (12 of ACCF, five of ACDF) (Table 1). All patients were monitored for $31.07 \pm 17.35$ months (range, $12-88.5$ months) after repeat surgery.

For each included patient, clinical and radiological data before the initial and repeat surgeries were evaluated in terms of : the interval between surgeries, locations of the main OPLL lesions for both initial and repeat surgeries, change in the level

Table 1. The distribution of surgical methods in the initial and repeat surgery

\begin{tabular}{lll}
\hline Group & \multicolumn{1}{c}{ Initial surgery } & Repeat surgery \\
\hline AP & ACCF 2 & Laminoplasty 7 \\
& ACDF 8 & Laminectomy plus fusion with screw fixation 3 \\
PA & Laminoplasty 5 & ACCF 12 \\
& Laminectomy alone 11 & ACDF 5 \\
& Laminectomy plus fusion with screw fixation 1 & \\
\hline
\end{tabular}

group AP : patients were approached anteriorly at the initial surgery and underwent posterior approach at the repeat surgery, group PA : patients were approached posteriorly at the initial surgery and underwent anterior approach at the repeat surgery, ACCF : anterior cervical corpectomy and fusion, ACDF : anterior cervical discectomy and fusion 
of main OPLL lesion between surgeries, OPLL type (classified into four types : continuous, segmental, mixed, and circumscribed $^{16}$ ), anterior-posterior diameter of the spinal canal and occupying ratio of OPLL at the main OPLL lesion, Cobb's angle of the entire cervical spine (C2-7), and the segmental angle at the main OPLL lesion.

\section{Radiologic evaluation (Fig. 1)}

Cobb's angle of the entire cervical curvature was measured and defined as the angle between the lines passing through the lower margin of the C2 and C7 vertebral bodies. The segmental angle was defined and measured by Cobb's method between the lines of the upper vertebral body's upper margin and the lower vertebral body's lower margin in the segment of interest. Cobb's and segmental angles were measured in the lateral X-ray or lateral view of computed tomography (CT) scan in neutral position. Lordosis was recorded as a positive value, and kyphosis was recorded as a negative value.

The OPLL occupying ratio was calculated as the maximum thickness of the OPLL to the anterior-posterior diameter of the spinal canal at the main OPLL lesion on CT scan ${ }^{5,16}$. In case of loss of the anterior or posterior structure of the spinal canal at the main OPLL lesion during initial surgery, the boundary of the dural sac anteriorly or posteriorly on T2 weighted image was regarded as the extent of the diameter.

\section{Functional evaluation}

The pre- and post-operative functional statuses related with repeat surgery in the all patients were measured using Nurick scale. Nurick scale is classified into 5 grades : 0 , signs or symptoms of root involvement but without evidence of spinal cord disease; 1 , signs of spinal cord disease but no difficulty in walking; 2, slight difficulty in walking that did not prevent fulltime employment; 3 , difficulty in walking that prevented fulltime employment or the ability to perform all housework but that was not severe enough to require someone else's help to walk; 4 , able to walk with someone else's help or with the aid of a frame; and 5 , chairbound or bedridden.

\section{Statistical analysis}

Student's $t$ test for parametric continuous variables and the Mann-Whitney test for non-parametric continuous variables were used to compare two population means. Paired $t$ test for parametric continuous variables and Wilcoxon signed rank test for non-parametric continuous variables were used to compare two population means where there were paired samples. Chi-square test was used to assess the relationship between categorical variables. Statistical analysis was carried out with SPSS version 20.0 software (SPSS Inc., Chicago, IL, USA), and probability values $<0.05$ were considered to be statistically significant.
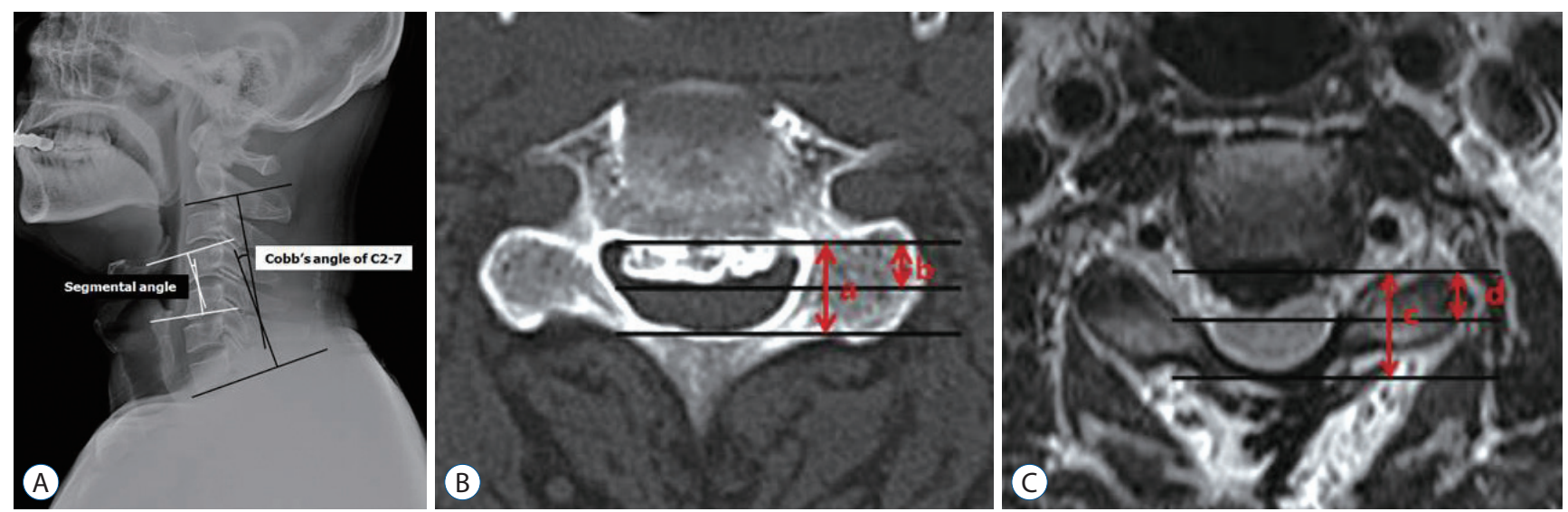

Fig. 1. Cobb's angle of the entire cervical curvature was measured as the angle between the lower margin of $C 2$ and $C 7$, and the segmental angle was defined and measured by Cobb's method between the lines of the upper vertebral body's upper margin and the lower vertebral body's lower margin in the segment of interest on a static neutral position (A). Lordosis was recorded as a positive value, and kyphosis was recorded as a negative value. The ossification of the posterior longitudinal ligament (OPLL) occupying ratio was calculated as the maximum thickness of the OPLL to the anteriorposterior diameter of the spinal canal at the main OPLL lesion on computed tomography scan (b/a) (B). In case of loss of the anterior or posterior structure of the spinal canal at the main OPLL lesion during initial surgery, the boundary of the dural sac anteriorly or posteriorly on $\mathrm{T} 2$ weighted image was regarded as the extent of the diameter $(\mathrm{d} / \mathrm{c})(\mathrm{C})$. 


\section{RESULTS}

\section{Comparison of the Groups AP and PA (Table 2)}

There were no statistically significant differences in mean age or sex distribution between groups AP and PA. Overall, participants were predominantly male $(9 / 10$ in group AP, 13/17 in group PA). The intervals between the initial and repeat surgeries were 102.80 \pm 60.08 months (group AP) and $61.44 \pm 43.04$ months (group PA). Among the 27 patients recruited for this study, those who underwent the initial surgery via the posterior approach (group PA) were more likely to experience a shorter interval between the initial and repeat surgeries than those with the initial surgery via the anterior approach (group AP) $(p<0.05)$. There was a statistically significant difference in the location of the main OPLL lesion causing renewed or newly developed neurological deterioration and leading to the repeat surgery between the two groups $(p<0.05)$. There was a tenden$c y$ that the site of the main OPLL lesion upon repeat surgery changed from that at the time of initial surgery in group AP (8/10). However, most main OPLL lesion sites from the initial surgery continued as a causative pathology leading to neurological deteriorations and repeat surgery in group PA (15/17).

Radiological parameters at the time of repeat surgery were measured and analyzed in the both groups. The mean OPLL occupying ratio at the main OPLL lesion leading to repeat surgery was larger in group AP $(52.22 \pm 11.51 \%)$ than in group PA $(44.67 \pm 12.82 \%)$, but this difference was not statistically significant $(p>0.05)$. The mean anterior-posterior diameter of the spinal canal at the main OPLL lesion was shorter in group AP $(10.75 \pm 0.79 \mathrm{~mm})$ than in group PA $(16.27 \pm 2.33 \mathrm{~mm} ; p<0.05)$. There were statistically significant differences in the Cobb's angle on $\mathrm{C} 2-7\left(7.44 \pm 5.54^{\circ}\right.$ in group $\mathrm{AP},-3.38 \pm 11.08^{\circ}$ in group PA) and segmental angle at the main OPLL lesion (2.71 $\pm 4.24^{\circ}$ in group $\mathrm{AP},-3.52 \pm 6.20^{\circ}$ in group $\left.\mathrm{PA} ; p<0.05\right)$. Group PA presented with more kyphotic curvature over the entire cervical sagittal alignment and the segmental angle at the site of the main OPLL lesion. Prominent kyphotic changes were noted in the Cobb's angle on C2-7 and segmental angles between the initial and repeat surgeries in group PA $(p<0.05)$. Of the patients in group PA, only one who had a decompressive laminectomy plus fusion with screw fixation showed more lordotic curvature in the Cobb's angle on C2-7 after the initial surgery (from 3.5 to 12.9). In this patient, another OPLL lesion at a different segment became the main site leading to repeat surgery. This lesion acted as the main pathology, and showed a progression of kyphosis in segmental angle (from -1.8 to -8.8).

Table 2. The comparison of clinical and radiological characteristics between the AP and PA groups

\begin{tabular}{|c|c|c|}
\hline Clinical and radiological characteristics & Group AP $(n=10)$ & Group PA $(n=17)$ \\
\hline Age & $61.70 \pm 6.18$ & $61.00 \pm 8.16$ \\
\hline $\operatorname{Sex}(M / F)$ & $9 / 1$ & $13 / 4$ \\
\hline \multicolumn{3}{|l|}{ OPLL type } \\
\hline Continuous & 2 & 4 \\
\hline Mixed & 6 & 11 \\
\hline Segmental & 2 & 1 \\
\hline Circumscribed & 0 & 1 \\
\hline Interval between the initial and repeat surgeries (months)* & $102.80 \pm 60.08$ & $61.44 \pm 43.04$ \\
\hline \multicolumn{3}{|l|}{ Change of the main OPLL lesion between surgeries* } \\
\hline$(-)$ & 2 & 15 \\
\hline$(+)$ & 8 & 2 \\
\hline Occupying ratio on the main OPLL lesion (at the time of repeat surgery) (\%) & $52.22 \pm 11.51$ & $44.67 \pm 12.82$ \\
\hline Canal anterior-posterior diameter on the main OPLL lesion (at the time of repeat surgery)* (mm) & $10.75 \pm 0.79$ & $16.27 \pm 2.33$ \\
\hline Cobb's angle on C2-7 (at the time of repeat surgery) ${ }^{*}\left({ }^{\circ}\right)$ & $7.44 \pm 5.54$ & $-3.38 \pm 11.08$ \\
\hline Segmental angle on the main OPLL lesion (at the time of repeat surgery) ${ }^{*}\left(^{\circ}\right)$ & $2.71 \pm 4.24$ & $-3.52 \pm 6.20$ \\
\hline
\end{tabular}

${ }^{*} p<0.05$. group AP : patients were approached anteriorly at the initial surgery and underwent posterior approach at the repeat surgery, group PA : patients were approached posteriorly at the initial surgery and underwent anterior approach at the repeat surgery, M : male, F : female, OPLL : ossification of the posterior longitudinal ligament, negative angle : kyphotic curve, positive angle : lordotic curve 


\section{Comparison of the subgroups LP and LN (Table 3)}

There was no statistically significant difference in mean age or sex distribution between the subgroups. Like the comparison between the groups AP and PA, more participants were male. The intervals between the initial and repeat surgeries were 56.60 \pm 74.61 months (subgroup LP) and 62.64 \pm 26.69 months (subgroup LN; $p>0.05$ ).

There were no statistically significant differences in Cobb's angle on C2-7 at the time of initial (4.58 $\pm 9.29^{\circ}$ in subgroup LP, $2.96 \pm 7.32^{\circ}$ in subgroup LN) and repeat $\left(-3.32 \pm 13.03^{\circ}\right.$ in subgroup LP, $-4.89 \pm 9.97^{\circ}$ in subgroup LN) surgery. The change of Cobb's angle on C2-7 after initial surgery between the subgroups $\left(-7.90 \pm 5.61^{\circ}\right.$ in subgroup LP, and $-7.86 \pm 7.48^{\circ}$ in subgroup LN; $p>0.05)$ also did not show significant difference. The segmental angle on the main OPLL lesion at the time of initial surgery reflected more kyphotic curvature in subgroup LN (-0.10 $\left.\pm 4.02^{\circ}\right)$ than in subgroup LP $\left(3.70 \pm 2.80^{\circ} ; p<0.05\right)$. However, there were no statistically significant differences between the subgroups in the segmental angle on the main OPLL lesion at the time of repeat surgery $\left(-1.78 \pm 6.79^{\circ}\right.$ in subgroup LP, $-3.85 \pm$ $6.22^{\circ}$ in subgroup LN; $\left.p>0.05\right)$ and the change of the segmental angle after initial surgery $\left(-5.48 \pm 5.69^{\circ}\right.$ in subgroup LP, -3.74 $\pm 2.76^{\circ}$ in subgroup LN; $p>0.05$ ).

\section{Functional status}

Most of the patients recovered neurologically after repeat surgery except two patients (one of group AP and one of group $\mathrm{PA})$. The improvements of cervical myelopathy of the patients based on Nurick scale were identified in Table 4. In group PA, there were more patients with advanced Nurick scale than group AP preoperatively $(p<0.05)$. However, the postoperative statuses were similar in the both groups.

\section{DISCUSSION}

Cervical myelopathy caused by OPLL can be treated via anterior or posterior approach, or both. Each has advantages and disadvantages. The decompression afforded by posterior surgery indirectly increases the space available for the spinal cord, so it can float away from the anteriorly compressing OPLL. Posterior surgery is usually preferred when more than two or three levels are affected by OPLL, because it is relatively easy and safe. The disadvantages of these procedures include risk of

Table 3. The comparison of clinical and radiological characteristics between the subgroups LP and LN in the group PA

\begin{tabular}{|c|c|c|}
\hline Clinical and radiological characteristics & $\begin{array}{l}\text { Subgroup LP } \\
\text { (laminoplasty) } \\
(n=5)\end{array}$ & $\begin{array}{c}\text { Subgroup LN } \\
\text { (laminectomy) } \\
(n=11)\end{array}$ \\
\hline Age & $61.00 \pm 3.54$ & $62.36 \pm 8.77$ \\
\hline $\operatorname{Sex}(M / F)$ & $4 / 1$ & $8 / 3$ \\
\hline \multicolumn{3}{|l|}{ OPLL type } \\
\hline Continuous & 1 & 3 \\
\hline Mixed & 3 & 7 \\
\hline Segmental & 0 & 1 \\
\hline Circumscribed & 1 & 0 \\
\hline Interval between the initial and repeat surgeries (months) & $56.60 \pm 74.61$ & $62.64 \pm 26.69$ \\
\hline Cobb's angle on C2-7 (at the time of initial surgery) $\left(^{\circ}\right)$ & $4.58 \pm 9.29$ & $2.96 \pm 7.32$ \\
\hline Cobb's angle on C2-7 (at the time of repeat surgery) $\left(^{\circ}\right)$ & $-3.32 \pm 13.03$ & $-4.89 \pm 9.97$ \\
\hline Changes of Cobb's angle on C2-7 (between initial and repeat surgery) $\left({ }^{\circ}\right)$ & $-7.90 \pm 5.61$ & $-7.86 \pm 7.48$ \\
\hline Segmental angle on the main OPLL lesion (at the time of initial surgery) ${ }^{*}\left({ }^{\circ}\right)$ & $3.70 \pm 2.80$ & $-0.10 \pm 4.02$ \\
\hline Segmental angle on the main OPLL lesion (at the time of repeat surgery) $\left(^{\circ}\right)$ & $-1.78 \pm 6.79$ & $-3.85 \pm 6.22$ \\
\hline Changes of segmental angle on the main OPLL lesion (between initial and repeat surgery) $\left(^{\circ}\right)$ & $-5.48 \pm 5.69$ & $-3.74 \pm 2.76$ \\
\hline
\end{tabular}

${ }^{*} p<0.05$. subgroup LP : patients underwent laminoplasty as a posterior decompression at the initial surgery, subgroup LN : patients underwent laminectomy alone as a posterior decompression at the initial surgery, group PA : patients were approached posteriorly at the initial surgery and underwent anterior approach at the repeat surgery, $\mathrm{M}:$ male, $\mathrm{F}$ : female, OPLL : ossification of the posterior longitudinal ligament, negative angle : kyphotic curve, positive angle : lordotic curve 
Table 4. The change of functional outcomes after repeat surgery

\begin{tabular}{|c|c|c|c|c|}
\hline & \multicolumn{2}{|c|}{ Group AP } & \multicolumn{2}{|c|}{ Group PA } \\
\hline & Preoperative & Postoperative & Preoperative & Postoperative \\
\hline \multicolumn{5}{|c|}{ Nurick scale } \\
\hline 0 & 0 & 5 & 0 & 8 \\
\hline 1 & 4 & 2 & 3 & 5 \\
\hline 2 & 1 & 2 & 7 & 3 \\
\hline 3 & 4 & 1 & 4 & 0 \\
\hline 4 & 1 & 0 & 3 & 1 \\
\hline 5 & 0 & 0 & 0 & 0 \\
\hline Average* & 2.20 & 0.90 & 2.41 & 0.88 \\
\hline
\end{tabular}

Nurick scale : 0 , signs or symptoms of root involvement but without evidence of spinal cord disease; 1 , signs of spinal cord disease but no difficulty in walking; 2 , slight difficulty in walking that did not prevent full-time employment; 3, difficulty in walking that prevented full-time employment or the ability to perform all housework but that was not severe enough to require someone else's help to walk; 4, able to walk with someone else's help or with the aid of a frame; 5 , chairbound or bedridden. ${ }^{*} p<0.05$

OPLL progression and insufficient correction of cervical kyphosis. On the other hand, anterior surgery directly decompresses the spinal cord by removing the OPLL. This allows for superior decompression and greater effectiveness than posterior surgery in maintaining or restoring cervical lordosis ${ }^{8,11)}$. However, anterior surgery has disadvantages including technical difficulty, inability to decompress cranial to C2, and higher risk of dural tears ${ }^{8,9)}$. Nevertheless, when the canal-occupying ratio of OPLL $>60 \%$, anterior decompression consistently outperforms posterior decompression ${ }^{4)}$.

Progression of the remaining lesions in multi-level OPLL continues after anterior or posterior decompression. The incidence of OPLL progression after laminoplasty has been reported at $70-73 \%$. This risk is greatest in younger patients $(<59$ years of age $)^{2,6)}$. The incidence of postoperative remaining OPLL progression at other levels after anterior surgery ranged from $36 \%$ to $64 \%{ }^{12)}$. The patho-mechanism of OPLL progression is still unclear, but mechanical stress has been implicated as an exacerbating factor ${ }^{15)}$. Postoperative changes in cervical alignment and segmental mobility might reflect dynamic instability, which could play a crucial role in the progression of OPLL and development of neurological deterioration. Many reports, including the present study, suggest OPLL progression is found more often in mixed-type OPLL ${ }^{3)}$.

Group AP exhibited a smaller anterior-posterior spinal canal diameter and larger OPLL occupying ratio at the main OPLL lesion compared with group PA at the time of repeat surgery. This indicates a direct effect of OPLL progression, which requires salvage surgery with additional posterior de- compression. Progression of remnant or newly developed OPLL at other segment plays an important role in the development of neurological symptoms after prior anterior surgery in patients with multi-level OPLL. The duration between the initial and repeat surgery in the AP group is longer than in the PA group, because the speed of OPLL progression may relatively slower than that of kyphotic change. Renewed or newly developed neurological symptoms could be seen when progression of remnant OPLL compromises more than $52.22 \pm 11.51 \%$ of the cervical canal in group AP.

Although the progression of OPLL is important, we think it is not a single and conclusive factor that makes repeat surgery necessary. In this study, we found that group PA has a wider anterior-posterior diameter of the spinal canal at the main OPLL lesion than group AP, and there tends to be a smaller OPLL occupying ratio at the main OPLL lesion in group PA than in group AP. In addition, the majority of main OPLL lesions in group PA continued as the main pathologies and led to repeat surgeries, in contrast to group AP. The most important trait related with repeat surgery in group PA was progression of kyphosis in the entire cervical curvature and segmental angle at the main OPLL lesion.

The occurrence of neurological symptoms in group PA was mainly caused by a progression of kyphosis in cervical curvature, even though there was the progression of OPLL. This mechanism differs from that of group AP, in which the majority of main OPLL lesions were removed during initial anterior surgery; progression of OPLL at a different segment led to neurological symptoms (Fig. 2). In group PA, neurological decom- 
pression had already been achieved in multiple segments during the initial posterior surgery, and progression of remnant OPLL at a different segment does not play as significant a role in development of neurological symptoms as in group AP (Fig. 3).

In previous study ${ }^{11}$, about half of the patients who underwent laminoplasty for myelopathy caused by cervical OPLL lost prior lordotic curvature later. Especially, the patients with $<5^{\circ}$ preoperative cervical lordosis showed significantly worse outcomes. Lee et al. ${ }^{7)}$ have reported that progression of cervical kyphosis in the patients who underwent laminectomy alone for multi-level OPLL was not statistically associated with the development of neurological symptom, even though there was
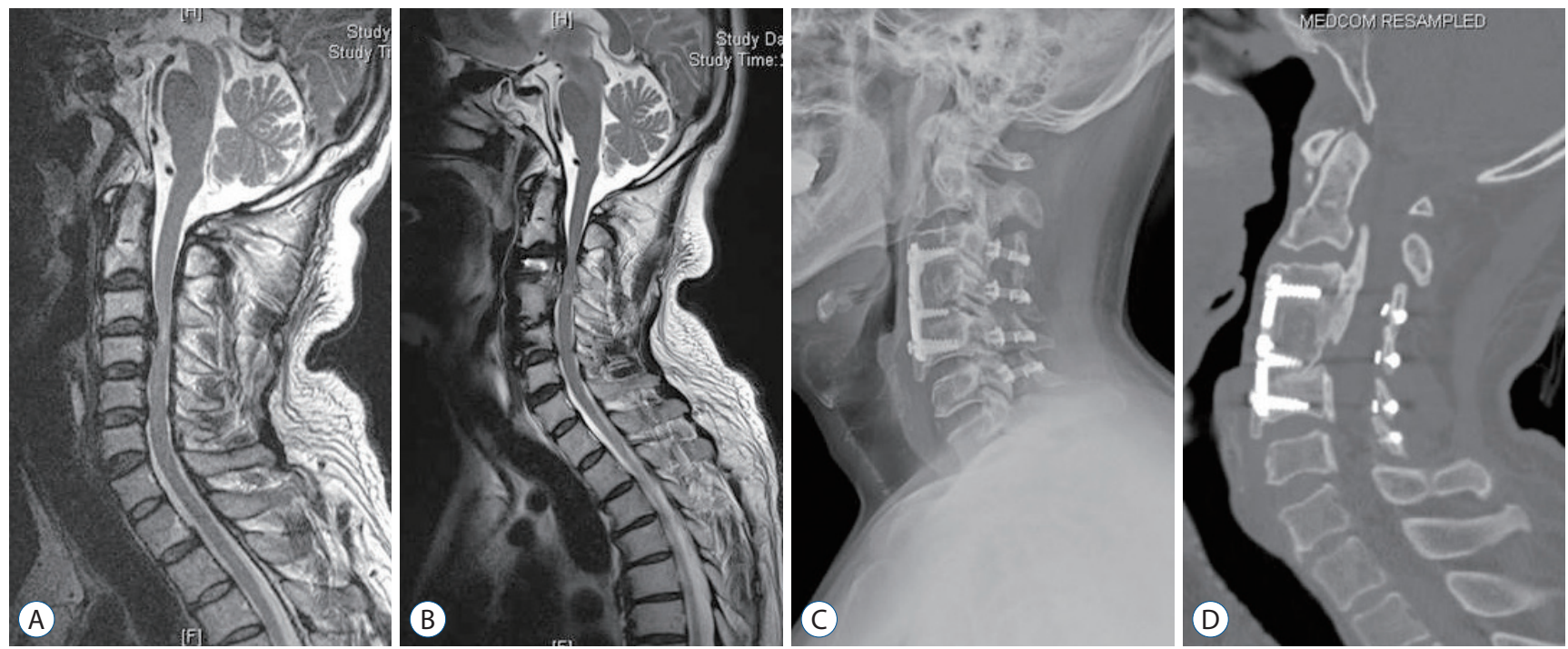

Fig. 2. Case 1 (group AP). A male patient, 55 years of age, complained myelopathy including gait disturbance and clumsy hand due to OPLL of C3-5. The symptoms were recovered completely after anterior cervical corpectomy and fusion on C3-5 with corpectomy of C4 (A). He presented of newly developed myelopathy originating from OPLL of C2-3 after 65.5 months (B). Laminoplasty on C3-6 with partial laminectomy of lower C2 was performed, the newly developed symptom disappeared with remaining mild shoulder pain (C and D). OPLL : ossification of the posterior longitudinal ligament.


Fig. 3. Case 2 (group PA). A male patient, 59 years of age, underwent laminectomy alone on C3-6 for OPLL of C3-6 causing myelopathy including gait disturbance and numbness of both arms (A). The symptoms was relieved and maintained for 30 months. However, he presented of gait disturbance and weakness of upper limbs again. The OPLL lesion of C4-5 continued as the main pathology causing initial myelopathy and newly developed symptoms with a progression of cervical kyphosis (the entire cervical Cobb's angle from 1.9 to -9.0) (B). Anterior cervical corpectomy and fusion on C3-5 with corpectomy of total C4 and upper C5 removing main OPLL lesion was performed, the patient shows improved gait disturbance with slight weakness of both hand grip (C and D). OPLL : ossification of the posterior longitudinal ligament; lordosis was recorded as a positive value, and kyphosis was recorded as a negative value. 
kyphosis progression of about $7^{\circ}$. The authors reported that OPLL has an effect on preventing progression of kyphosis, and that laminectomy alone could be an affordable surgical method as a posterior decompression in OPLL. However, our results revealed a progression of kyphosis by more than a mean of $7^{\circ}$ in the LP and LN subgroups and development of neurological symptoms. This difference may be originated from the preoperative cervical curvature, our participants' result showed more kyphotic curvature $\left(4.58 \pm 9.29^{\circ}\right.$ in subgroup LP, $2.96 \pm 7.32^{\circ}$ in subgroup LN) than that study $\left(11.3 \pm 7.0^{\circ}\right)$.

One patient in our study experienced newly developed neurological deterioration due to segmental kyphosis after laminectomy and fusion. This segmental kyphosis may have developed during the fusion process. When multi-segment cervical OPLL patients with relatively straight cervical spines are treated by posterior surgery, however, we believe posterior fusion with screw fixation is required to avoid recurrence of neurological symptoms due to progression of kyphosis.

\section{CONCLUSION}

The reasons for renewed or newly developed neurological deterioration in patients with multi-level OPLL depend on the initial surgery type. The main causative factors leading to neurological deterioration and repeat surgery are progression of remnant OPLL at the other segment in group AP, and kyphotic change in the cervical curve in group PA.

\section{CONFLICTS OF INTEREST}

The authors have no financial conflicts of interest.

\section{INFORMED CONSENT}

This type of study does not require informed consent.

\section{References}

1. An HS, Al-Shihabi L, Kurd M : Surgical treatment for ossification of the posterior longitudinal ligament in the cervical spine. J Am Acad Or- thop Surg $22:$ 420-429, 2014

2. Chiba K, Yamamoto I, Hirabayashi H, Iwasaki M, Goto H, Yonenobu K, et al. : Multicenter study investigating the postoperative progression of ossification of the posterior longitudinal ligament in the cervical spine: a new computer-assisted measurement. J Neurosurg Spine 3: 17-23, 2005

3. Hori T, Kawaguchi $Y$, Kimura $T$ : How does the ossification area of the posterior longitudinal ligament thicken following cervical laminoplasty? Spine (Phila Pa 1976) 32 : E551-E556, 2007

4. Iwasaki M, Okuda S, Miyauchi A, Sakaura H, Mukai Y, Yonenobu K, et al. : Surgical strategy for cervical myelopathy due to ossification of the posterior longitudinal ligament: Part 1: clinical results and limitations of laminoplasty. Spine (Phila Pa 1976) 32 : 647-653, 2007

5. Kato Y, Iwasaki M, Fuji T, Yonenobu K, Ochi T : Long-term follow-up results of laminectomy for cervical myelopathy caused by ossification of the posterior longitudinal ligament. J Neurosurg 89 : 217-223, 1998

6. Kawaguchi $Y$, Kanamori M, Ishihara H, Nakamura H, Sugimori K, Tsuji $H$, et al. : Progression of ossification of the posterior longitudinal ligament following en bloc cervical laminoplasty. J Bone Joint Surg Am 83-A : 1798-1802, 2001

7. Lee SE, Chung CK, Jahng TA, Kim HJ : Long-term outcome of laminectomy for cervical ossification of the posterior longitudinal ligament. J Neurosurg Spine $18: 465-471,2013$

8. Mizuno J, Nakagawa H : Outcome analysis of anterior decompressive surgery and fusion for cervical ossification of the posterior longitudinal ligament: report of 107 cases and review of the literature. Neurosurg Focus 10 : E6, 2001

9. Mizuno J, Nakagawa H, Matsuo N, Song J : Dural ossification associated with cervical ossification of the posterior longitudinal ligament: frequency of dural ossification and comparison of neuroimaging modalities in ability to identify the disease. J Neurosurg Spine 2 : 425-430, 2005

10. Onji Y, Akiyama H, Shimomura Y, Ono K, Hukuda S, Mizuno S : Posterior paravertebral ossification causing cervical myelopathy. A report of eighteen cases. J Bone Joint Surg Am 49 : 1314-1328, 1967

11. Sakai K, Okawa A, Takahashi M, Arai Y, Kawabata S, Enomoto M, et al. : Five-year follow-up evaluation of surgical treatment for cervical myelopathy caused by ossification of the posterior longitudinal ligament: a prospective comparative study of anterior decompression and fusion with floating method versus laminoplasty. Spine (Phila Pa 1976) 37 : 367-376, 2012

12. Satomi K, Ogawa J, Ishii Y, Hirabayashi K : Short-term complications and long-term results of expansive open-door laminoplasty for cervical stenotic myelopathy. Spine J $1:$ 26-30, 2001

13. Smith ZA, Buchanan CC, Raphael D, Khoo LT : Ossification of the posterior longitudinal ligament: pathogenesis, management, and current surgical approaches. A review. Neurosurg Focus 30 : E10, 2011

14. Tani T, Ushida T, Ishida K, lai H, Noguchi T, Yamamoto H : Relative safety of anterior microsurgical decompression versus laminoplasty for cervical myelopathy with a massive ossified posterior longitudinal ligament. Spine (Phila Pa 1976) 27 : 2491-2498, 2002

15. Tsukamoto N, Maeda T, Miura H, Jingushi S, Hosokawa A, Harimaya K, et al. : Repetitive tensile stress to rat caudal vertebrae inducing cartilage 
formation in the spinal ligaments: a possible role of mechanical stress in the development of ossification of the spinal ligaments. J Neurosurg Spine 5 : 234-242, 2006
16. Tsuyama $N$ : Ossification of the posterior longitudinal ligament of the spine. Clin Orthop Relat Res (184) : 71-84, 1984 